ISSN 1980-5098

\title{
AVALIAÇÃO DAS PROPRIEDADES FÍSICO-MECÂNICAS DE PAINÉIS DE CASCA DE ARROZ E ADESIVO TANINO-FORMALDEÍDO
}

\author{
EVALUATION OF PHYSICAL AND MECHANICAL PROPERTIES OF RICE HUSK PANELS \\ GLUED WITH TANNIN-FORMALDEHYDE
}

\author{
Joel Telles de Souza ${ }^{1}$ Walmir Marques de Menezes ${ }^{1}$ Clovis Roberto Haselein ${ }^{2}$ Talita Baldin ${ }^{1}$ \\ Rafael da Rosa Azambuja ${ }^{3}$ Weslley Wilker Corrêa Morais ${ }^{4}$
}

\begin{abstract}
RESUMO
A presente investigação tem como objetivo avaliar o uso da casca de arroz para a fabricação de chapas aglomeradas, coladas com tanino-formaldeído. Para tanto, foram utilizadas cascas de arroz na forma natural ou processadas em moinho de martelo, compactadas a diferentes massas específicas $(0,65,0,95$ e $1,15 \mathrm{~g} / \mathrm{cm}^{3}$ ) e aplicando-se teores de adesivos de 7, 10 e $13 \%$ (com base no peso seco das partículas), totalizando 54 chapas. A prensagem foi realizada em prensa hidráulica a $30 \mathrm{kgf} / \mathrm{cm}^{2}$, por 8 minutos, a uma temperatura de $180^{\circ} \mathrm{C}$. Os ensaios físicos realizados foram teor de umidade, massa específica, absorção d'água e inchamento em espessura; e mecânicos, tais como flexão estática, arrancamento de parafuso e ligação interna. De modo geral, a utilização da casca de arroz proporcionou uma redução na resistência física e mecânica das chapas, em relação às produzidas com partículas de madeira encontradas na literatura. Todos os tratamentos ficaram abaixo dos valores mínimos requeridos pelas normas de comercialização para os ensaios físicos e mecânicos, somente os de $13 \%$ de adesivo apresentaram requisitos satisfatórios quanto à estabilidade dimensional. Os resultados indicam que o material não pode ser utilizado em condições que exijam grandes esforços estruturais.
\end{abstract}

Palavras-chave: beneficiamento do arroz; resíduos; propriedades físico-mecânicas; estabilidade dimensional.

\section{ABSTRACT}

Rice husk, waste produced by processing of rice, has become a problem of environmental, economic and public health. As a result, this research aims to explore the potential of these underutilized resources in Brazil in the development of particleboard glued with tannin-formaldehyde. Therefore, panels were manufactured with rice husk compacted at different density $\left(0.65,0.95\right.$ and $\left.1.15 \mathrm{~g} / \mathrm{cm}^{3}\right)$, levels of adhesives $(7,10$ and $13 \%$ ) and particles types (natural and processed), totaling 54 panels. The pressing was carried in hydraulic press at $30 \mathrm{kgf} / \mathrm{cm}^{2}$ for 8 minutes at $180^{\circ} \mathrm{C}$ of temperature. The physical tests performed were moisture content, density, water absorption and thickness swelling while mechanical properties evaluated were static bending, screw withdrawal and internal bond. In general, the use of rice hulls prouced a reduction in strength

1 Engenheiro Florestal, doutorando em Tecnologia de Produtos Florestais. Departamento de Ciências Florestais, Centro de Ciências Rurais, Universidade Federal de Santa Maria, Av. Roraima, 1000, CEP 97105-900, Santa Maria (RS), Brasil.joel.telles@yahoo.com.br/walmirmenezessm@hotmail.com/talita.baldin@hotmail.com

2 Engenheiro Florestal, Phd., Professor Associado do Departamento de Ciências Florestais, Centro de Ciências Rurais, Universidade Federal de Santa Maria, Av. Roraima, 1000, CEP 97105-900, Santa Maria (RS), Brasil. clovis.haselein@ufsm.br

3 Engenheiro Florestal, Mestrando do Programa de Pós Graduação em Engenharia Florestal, Universidade Federal do Paraná, Av. Lothário Meissner, 632 - Jardim Botânico, CEP 80210-170, Curitiba (PR), Brasil. rafael.r.azambuja@gmail.com

4 Engenheiro Florestal, M.Sc., Professor titular do Curso de Engenharia Florestal, Universidade Estadual de Roraima, Rua 7 de Setembro, 231, Canarinho. CEP 69306-530, Boa Vista (RR). Brasil. weslley_eng@yahoo.com.br

Recebido para publicação em 16/06/2014 e aceito em 02/03/2016 
when compared to those made with wood particles. All particleboard were below the minimum standards in physical and mechanical properties; only the panels with $13 \%$ resin provided minimum values required by market standards regarding dimensional stability. The results indicate that the material cannot be used in conditions requiring great structural efforts.

Keywords: rice hulls processing; waste; Physical-mechanical properties; dimensional stability.

\section{INTRODUÇÃO}

A produção de resíduos na cultura do arroz está relacionada à palha e, principalmente, à casca, que corresponde a aproximadamente $20 \%$ do peso do arroz. Composta de sílica, a casca implica em três problemas principais: primeiro, de ordem ambiental, esses resíduos, por serem de difícil decomposição, permanecem no solo por vários anos, causando agressão ao meio ambiente. Segundo, de saúde pública, já que a sua queima, por vezes, a céu aberto, gera produtos tóxicos ao homem. O terceiro problema é de ordem econômica, já que o desuso do material constitui uma forma de desperdício, uma vez que a ele poderia ser agregado valor para então servir de incremento na renda do produtor.

No Rio Grande do Sul, comenta Melo (2009), tem-se um volume expressivo desse resíduo concentrado na Metade Sul do estado, o que facilita a logística de utilização. Estimando-se que, na safra de 2014/2015, o Estado produziu 8,72 milhões de toneladas de arroz base casca, ter-se-ia, aproximadamente, dois milhões de toneladas de casca de arroz anualmente (INSTITUTO RIO GRANDENSE DO ARROZ, 2015; SOCIEDADE SUL BRASILEIRA DE ARROZ IRRIGADO, 2014). Uma fábrica de chapas aglomeradas de médio porte consome, por ano, de 200 a 500 mil toneladas de matéria-prima (madeira). Então, esse volume seria suficiente para suprir a demanda de mais de uma unidade industrial. Nesse sentido, está se buscando desenvolver formas de aproveitamento desse material na fabricação de chapas aglomeradas, para evitar ou diminuir o problema ambiental, de saúde pública e econômico.

A produção de painéis aglomerados a partir de resíduos agroindustriais, conforme mencionado por Mendes et al. (2010), proporciona valor agregado ao resíduo, bem como, mostra-se uma importante ferramenta para suprir a crescente demanda na indústria de painéis de madeira, além de possibilitar sua expansão, diminuir a utilização de materiais lenhosos e, consequentemente, a pressão sobre as florestas, e ainda reduzir custos de produção dos painéis, tornando-os ainda mais competitivos no cenário econômico.

Por outro lado, colagens com adesivos à base de tanino têm-se mostrado eficientes, com propriedades muitas vezes superiores aos da ureia-formaldeído, principal adesivo empregado no Brasil atualmente. Possui como vantagens ser de fonte renovável e produzido em escala industrial no Rio Grande do Sul. Carneiro et al. (2009) comentam sobre a importância de pesquisas para o desenvolvimento de adesivos tânicos como alternativas para a indústria de painéis, visto que o custo da aquisição de adesivos sintéticos representa um componente de peso no valor final do produto e, também, pelo alto percentual de formaldeído presente nos adesivos de ureia-formaldeído, principal aglutinante utilizado na produção de chapas.

Dentro desse escopo, o presente trabalho tem como objetivo avaliar o aproveitamento de casca de arroz (Oryza sativa L) para a fabricação de chapas aglomeradas, coladas com adesivo à base de tanino-formaldeído, possibilitando avaliar as propriedades físico-mecânicas desses novos compósitos. Cabe salientar que os resultados apresentados nesse trabalho, além de contribuírem com o desenvolvimento científico, encorajam novas possibilidades de pesquisas e uso dos resíduos provenientes da produção de arroz.

\section{MATERIAL E MÉTODOS}

\section{Obtenção e preparo da matéria-prima}

A casca de arroz utilizada nesta pesquisa foi procedente da Indústria de Beneficiamento de Arroz Cerealista De David Ltda., localizada no bairro Camobi, município de Santa Maria - RS. O material foi levado para o Laboratório de Produtos Florestais (LPF), da Universidade Federal de Santa Maria (UFSM), no qual passou pelo processo de preparação, que consistiu em passagem por moinho de martelo com 
peneira de orifícios de 5,0 mm de diâmetro (partículas processadas) e sem passagem por moinho de martelo (partículas naturais).

Após, todo material, tanto natural como processado, passou por peneira de malha de $1,0 \mathrm{~mm} \mathrm{x}$ $1,0 \mathrm{~mm}$ para descarte das partículas mais finas e do pó, sendo aproveitado o material que não passou pela malha. Posteriormente, as partículas foram secas em estufa a, aproximadamente, $50^{\circ} \mathrm{C}$ por um período que variou entre 12 a 24 horas, até um teor de umidade de 3\%, ideal para confecção das chapas.

O tanino da acácia-negra (Acacia mearnsii De Wild), na forma de pó, foi doado pela empresa SETA, localizada no município de Estância Velha - RS. Para produção do adesivo em laboratório, o tanino foi hidratado 24 horas antes da formulação, através do preparo de uma solução em água com aproximadamente $50 \%$ de sólidos. Posteriormente, foi adicionado hidróxido de sódio $(\mathrm{NaOH})$ até que o $\mathrm{pH} 8$ fosse atingido, sendo que, para esta medição, utilizou-se de pHmetro. O formaldeído foi adicionado apenas no momento da aplicação do adesivo, sendo utilizado na proporção de 10\% (base peso seco do tanino).

\section{Fabricação das chapas}

Para o monitoramento do teor de umidade das partículas de casca de arroz, após a secagem, foram separadas 5 amostras, secas em estufa a $103^{\circ} \mathrm{C}$ até massa constante. As partículas foram então misturadas com o adesivo tanino-formaldeído em um tambor rotativo. A aplicação do adesivo foi por meio de uma pistola acionada por compressor de ar com pressão de $8 \mathrm{Kgf} / \mathrm{cm}^{2}$ e vazão de $50 \mathrm{~g} / \mathrm{min}$. Para facilitar a aplicação do tanino-formaldeído, foi adicionada a quantidade de água necessária para ajustar o teor de umidade do colchão (TUc) em, aproximadamente, $20 \%$. O colchão foi formado manualmente em uma caixa formadora com dimensões de $40 \mathrm{~cm}$ x $40 \mathrm{~cm}$ x $20 \mathrm{~cm}$ e pré-prensado em prensa manual à temperatura ambiente por, aproximadamente, 1 minuto.

Após a prensagem a frio, o colchão de partículas foi retirado da caixa formadora e coberto com uma folha de papel-vegetal e uma chapa de aço galvanizada. Em seguida, foi então levado à prensa hidráulica, da marca OMECO, com capacidade para aplicação de carga de 100 toneladas e pratos com dimensões de $60 \mathrm{~cm} \times 60 \mathrm{~cm}$.

Nesse sentido, as chapas foram confeccionadas com dimensões de $40 \mathrm{~cm}$ x $40 \mathrm{~cm}$ x 0,95 cm, com pressão específica de $30 \mathrm{kgf} / \mathrm{cm}^{2}$ e temperatura dos pratos ajustada para $180^{\circ} \mathrm{C}$. A espessura de $0,95 \mathrm{~cm}$ foi definida por gabaritos de barras de aço, as quais foram colocadas nas laterais do colchão, limitando o fechamento da prensa. O tempo total de prensagem aplicado para promover a evaporação da água e a cura do adesivo foi de 8 minutos.

Após a prensagem, as chapas foram identificadas, lixadas e tiveram suas bordas aparadas, passando para as dimensões finais de $39 \mathrm{~cm}$ x $39 \mathrm{~cm}$ x $0,95 \mathrm{~cm}$. Estas foram dispostas em câmara climatizada $\left(20^{\circ} \mathrm{C}\right.$ e $65 \%$ de umidade relativa) até massa constante.

\section{Delineamento experimental}

O experimento, conforme Tabela 1, foi realizado com as seguintes avaliações: teor de adesivos (TA): 7, 10 e 13\% (com base no peso seco das partículas); massa específica (ME) das chapas: 0,65, 0,95 e $1,15 \mathrm{~g} / \mathrm{cm}^{3}$; preparo das partículas: Natural $(\mathrm{N})$ e Processada $(\mathrm{P})$ em moinho de martelo. Estas combinações perfizeram um total de 18 tratamentos, sendo que, para cada um destes tratamentos avaliados, foram feitas 3 repetições, totalizando, portanto, 54 chapas confeccionadas a partir do resíduo de casca de arroz.

\section{Ensaios realizados e análise dos resultados}

Para avaliação da qualidade das chapas produzidas, foram retirados corpos de prova para determinação das propriedades físicas (teor de umidade; massa específica; absorção d'água e inchamento em espessura) e mecânicas (flexão estática; arrancamento de parafuso e ligação interna), adotando-se as recomendações da norma ASTM D 1037 (1998).

Os resultados dos ensaios físicos e mecânicos foram submetidos à análise de regressão linear 
TABELA 1: Delineamento experimental.

TABLE 1: Experimental layout.

\begin{tabular}{cccc}
\hline Tratamento & TA $(\%)$ & ME $\left(\mathrm{g} / \mathrm{cm}^{3}\right)$ & Partícula \\
\hline 1 & 7 & 0,65 & $\mathrm{~N}$ \\
2 & 7 & 0,65 & $\mathrm{P}$ \\
3 & 7 & 0,95 & $\mathrm{~N}$ \\
4 & 7 & 0,95 & $\mathrm{P}$ \\
5 & 7 & 1,15 & $\mathrm{~N}$ \\
6 & 7 & 1,15 & $\mathrm{P}$ \\
7 & 10 & 0,65 & $\mathrm{~N}$ \\
8 & 10 & 0,65 & $\mathrm{P}$ \\
9 & 10 & 0,95 & $\mathrm{~N}$ \\
10 & 10 & 0,95 & $\mathrm{P}$ \\
11 & 10 & 1,15 & $\mathrm{~N}$ \\
12 & 10 & 1,15 & $\mathrm{P}$ \\
13 & 13 & 0,65 & $\mathrm{~N}$ \\
14 & 13 & 0,65 & $\mathrm{P}$ \\
15 & 13 & 0,95 & $\mathrm{~N}$ \\
16 & 13 & 0,95 & $\mathrm{P}$ \\
17 & 13 & 1,15 & $\mathrm{~N}$ \\
18 & 13 & 1,15 & $\mathrm{P}$ \\
\hline
\end{tabular}

Em que: $\mathrm{TA}=$ teor de adesivos; $\mathrm{ME}$ = massa específica; $\mathrm{N}$ = partícula natural; $\mathrm{P}$ = partícula processada.

múltipla, a 5\% de probabilidade de erro. A seleção dos melhores modelos foi realizada com base nos menores valores de $\mathrm{P}$ e Syx (erro padrão da estimativa), nos maiores valores de F e $\mathrm{R}^{2}$ aj (coeficiente de determinação ajustado) e através da análise visual dos gráficos dos resíduos.

\section{RESULTADOS E DISCUSSÃO}

\section{Propriedades físicas das chapas}

\section{Massa específica e teor de umidade}

A massa específica nominal (MEn) foi de $0,65 \mathrm{~g} / \mathrm{cm}^{3}$ para as chapas de média densidade e 0,95 $\mathrm{g} / \mathrm{cm}^{3}$ e $1,15 \mathrm{~g} / \mathrm{cm}^{3}$ para as de alta densidade. Entretanto, foram encontrados valores de massa específica observada (MEo) variando entre 0,628 e $0,640 \mathrm{~g} / \mathrm{cm}^{3}, 0,925$ e $0,935 \mathrm{~g} / \mathrm{cm}^{3}, 1,120$ e $1,130 \mathrm{~g} / \mathrm{cm}^{3}$, respectivamente. A variação pode ser considerada comum e atribuída ao processo manual de manufatura das chapas, especialmente nas fases de montagem do colchão e/ou na adição de adesivos e outros aditivos, como já havia sido anteriormente reportado por Hillig, Haselein e Santini (2002) e Dacosta (2005).

O teor de umidade de equilíbrio (TUeq) das amostras variou entre 10,20 e 10,38\%. Percebe-se que o acréscimo da massa específica e o teor de adesivo (TA) nas chapas de casca de arroz não influenciaram na sua umidade de equilíbrio. Todos os tratamentos apresentaram um padrão de variação semelhante, não sendo observada diferença significativa.

\section{Absorção d'água}

As chapas fabricadas com casca de arroz processada tiveram, na maioria dos tratamentos, maior taxa de absorção d'água em 24 horas de imersão (AA24H), se comparadas às de partículas naturais. Isso ocorre devido à maior superfície de contato das partículas processadas. Entretanto, esta diferença de absorção entre 
os tipos de partículas é menor nesse tempo de imersão (Figura 1).

Como era esperado, a absorção d'água diminuiu com o aumento da massa específica, o que pode ser explicado pela diminuição dos espaços vazios em amostras mais densas (HILLIG; HASELEIN; SANTINI 2002; DACOSTA, 2005; PEDRAZZI, 2005). Quanto maior o teor de adesivo menor a absorção d'água, portanto, os tratamentos com $13 \%$ de adesivo e $1,15 \mathrm{~g} / \mathrm{cm}^{3}$ de massa específica tiveram os melhores valores: $23,30 \%$ para partículas naturais e $29,61 \%$ para processadas. Valor semelhante ao verificado por Melo (2009), que encontrou $20 \%$ de absorção para chapas com $100 \%$ de partículas naturais de casca de arroz e superior à literatura brasileira com chapas de madeira.

A absorção após 2 e 24 horas encontrada para a casca de arroz é notavelmente inferior àquela verificada em chapas de madeira aglomerada produzidas com distintas espécies florestais, Pinus elliottii e Eucalyptus dunnii (IWAKIRI et al., 1996), Pinus sp e Mimosa scabrella (IWAKIRI et al., 1995). Os resultados podem ser atribuídos aos altos teores de sílica em sua composição, sendo uma característica positiva para absorção d'água. Contrariamente, os valores obtidos nessa pesquisa, são ligeiramente superiores à casca de café (ARAÚJO et al., 2014) e à casca de aveia (VARANDA, 2013).

Quando comparado à absorção das chapas nos dois tempos de imersão, é possível notar a semelhança de comportamento das equações ajustadas. Assim como no tempo de 2 horas, a imersão em 24 horas diminui com o aumento da massa específica. Os resultados encontrados mantêm similaridade com Hillig, Haselein e Santini (2002), Dacosta et al. (2005) e Pedrazzi et al. (2005), embora os autores não tenham utilizado os mesmos materiais na confecção dos painéis, observou-se relação direta entre a massa especifica
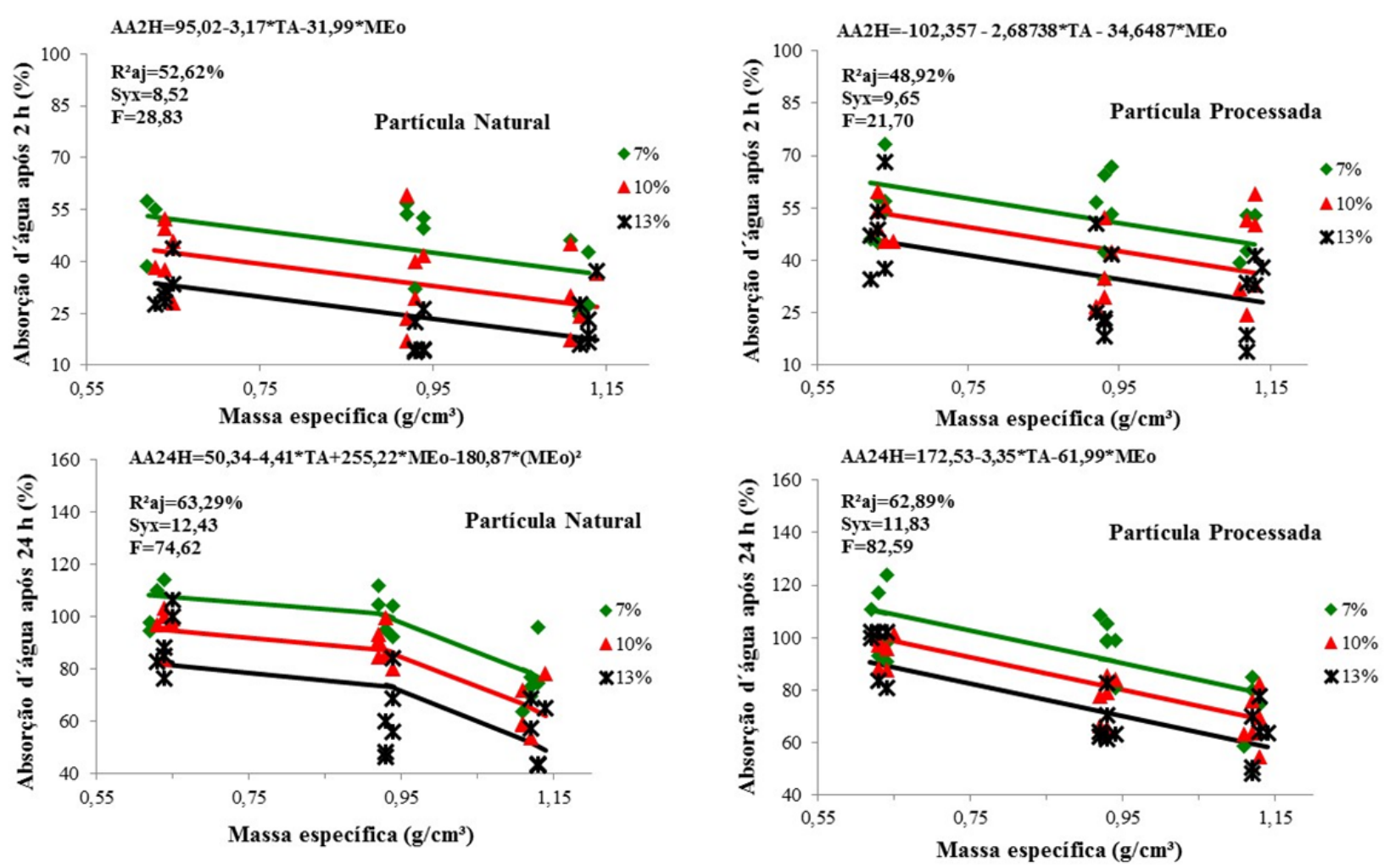

FIGURA 1: Valores de absorção d'água em 2 e 24 horas de imersão (AA2H e AA24H), observados e ajustados em função da massa específica, para chapas de partículas naturais e processadas, com 3 teores de adesivos.

FIGURE 1: Values of water absorption 2 and 24 hours immersion (AA2H e AA24H) observed and adjusted as a function of density, for particleboard of natural and processed particles, with 3 levels of adhesives. 
e a absorção. Além disso, houve diferença significativa a 5\% de probabilidade de erro para os 3 teores de adesivo, sendo que, quanto maior essa característica, menor a absorção d'água, mantendo novamente a conformidade com pressupostos de Iwakiri et al. (2000), Dacosta et al. (2005) e Pedrazzi et al. (2005).

\section{Inchamento em espessura}

Os valores médios de inchamento em espessura, alcançados nos períodos de 2 e 24 horas de imersão (IE2H e IE24H, respectivamente), variaram de 8,44 a 25,71\% para IE2H e 23,69 a 66,22\% para IE24H (Figura 2).

O inchamento em espessura nas chapas confeccionadas com partículas naturais foi menor naquelas com massa específica de 0,95 e $1,15 \mathrm{~g} / \mathrm{cm}^{3}$, no entanto, nas chapas com partículas processadas, o inchamento após 2 horas de imersão manteve-se constante em $0,65,0,95$ e $1,15 \mathrm{~g} / \mathrm{cm}^{3}$.

Para ambos os tipos de partículas houve diferença significativa a $5 \%$ de probabilidade de erro, quanto ao teor de adesivos, exceto o tanino de 7 e 10\% para partículas naturais, que não diferiram estatisticamente. Os demais seguiram a mesma tendência observada na absorção d'água, ou seja, quanto maior o teor do adesivo tânico menor o inchamento em espessura.

A norma brasileira ABNT NBR 14810-1 (2006) estabelece que o inchamento em espessura após 2 horas seja no máximo de $8 \%$. Todos os valores foram superiores ao exigido pela ABNT. Entretanto, as chapas com partículas naturais, $13 \%$ de tanino e $1,15 \mathrm{~g} / \mathrm{cm}^{3}$ de massa específica, chegaram muito próximo ao estabelecido pela norma brasileira $(8,44 \%)$. Outras chapas também apresentaram um bom potencial ao inchamento em espessura após 2 horas, principalmente as de $13 \%$ de adesivo.

Melo (2009) encontrou 14,71\% de inchamento após 2 horas, com partículas de casca de arroz
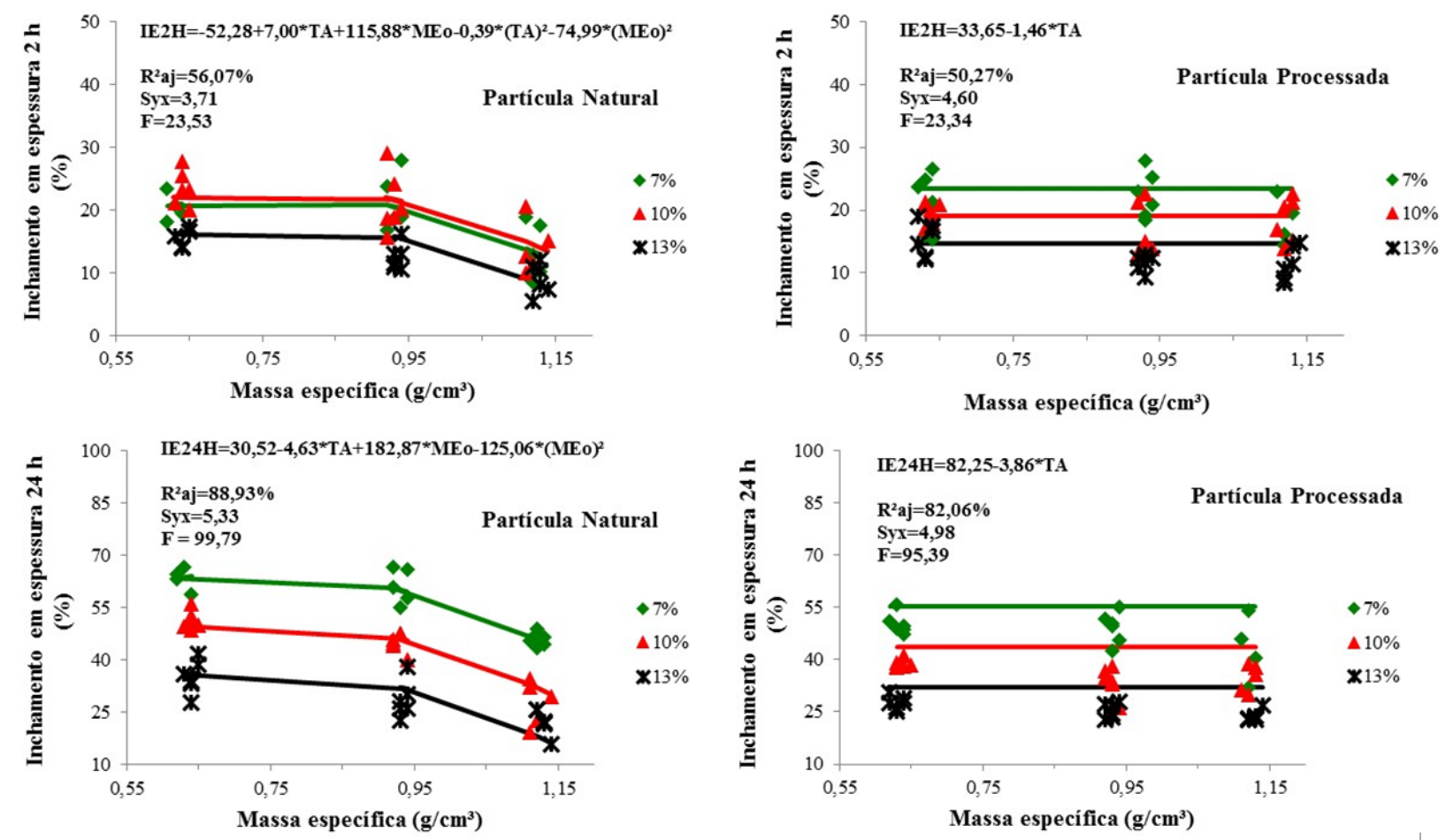

FIGURA 2: Valores de inchamento em espessura em 2 e 24 horas de imersão (IE2H e IE24H) observados e ajustados em função da massa específica, para chapas de partículas naturais e processadas, com 3 teores de adesivos.

FIGURE 2: Values of thickness swelling 2 and 24 hours immersion (IE2H e IE24H) observed and adjusted as a function of density, for particleboard of natural and processed particles, with 3 levels of adhesives. 
naturais, $0,65 \mathrm{~g} / \mathrm{cm}^{3}$ de massa específica e $8 \%$ de adesivo, valor um pouco inferior ao tratamento de características semelhantes ao do presente estudo (20,47\%). Esta diferença pode ter ocorrido pelo menor teor de adesivo deste tratamento $(7 \%)$ e pela não utilização da emulsão de parafina, já que esta melhora os valores de absorção e inchamento das chapas. Além disso, Pizzi, Scharfetter e Kes (1981) afirmam que a qualidade de adesivos depende muito de sua formulação, do material de origem e de tratamentos para melhoria de sua eficiência.

A equação ajustada para chapas de partículas naturais mostra uma baixa declividade da reta entre a massa específica de 0,65 a $0,95 \mathrm{~g} / \mathrm{cm}^{3}$, mas que se acentua entre 0,95 a $1,15 \mathrm{~g} / \mathrm{cm}^{3}$. Sendo assim, estes resultados mantêm a similaridade de comportamento com Albuquerque e Iwakiri (2005) e Morais (2011). Enquanto as chapas com partículas processadas não apresentaram diferença significativa, a 5\% de probabilidade de erro, entre inchamento em espessura após 2 horas e massa específica.

Semelhante aos resultados obtidos no inchamento em 2 horas de imersão, para o período de 24 horas, nos três teores de adesivos analisados, também houve significância a $95 \%$ de confiabilidade, sendo maiores os valores de inchamento quanto maior a porcentagem de tanino.

Os melhores valores encontrados de inchamento em espessura após 24 horas para chapas de partículas naturais e processadas foram 23,83 e 23,69\%, respectivamente. A norma DIN 68761 (1961) estabelece que o inchamento em espessura, após 24 horas, seja no máximo de $15 \%$. Sendo assim, todos os tratamentos estão com valores superiores ao estabelecido. No entanto, a norma ANSI 208.1 (1987) considera aceitável o percentual de até $35 \%$, valor atingido por todas as chapas (natural e processada) com $13 \%$ e $10 \%$ de adesivo e $1,15 \mathrm{~g} / \mathrm{cm}^{3}$ de massa específica, também para ambas as partículas.

\section{Propriedades mecânicas das chapas}

\section{Flexão estática}

Os valores médios de módulo de elasticidade (MOE) variaram entre 1115,60 e 8991,60 kgf/ $\mathrm{cm}^{2}$, enquanto o módulo de ruptura (MOR) obteve variação de 29,01 a 66,27 kgf/ $\mathrm{cm}^{2}$ (Figura 3).

Corroborando os resultados amplamente mencionados na literatura, o melhor desempenho no quesito resistência foi observado para as partículas com maior massa específica 1,15 g/ $/ \mathrm{cm}^{3}\left(66,27 \mathrm{kgf} / \mathrm{cm}^{2}\right)$. Porém, o valor foi inferior à norma americana de comercialização, ANSI A 208.1 (1987), para chapas de média $\left(0,64\right.$ a $\left.0,80 \mathrm{~g} / \mathrm{cm}^{3}\right)$ e alta densidade $\left(0,80\right.$ a $\left.1,12 \mathrm{~g} / \mathrm{cm}^{3}\right)$ do tipo 1 , que admite como valor mínimo requerido 112 e $168 \mathrm{kgf} / \mathrm{cm}^{2}$, respectivamente, para média e alta massa específica.

Melo (2009) encontrou um MOR médio de 37,44 Kgf $/ \mathrm{cm}^{2}$ para $0,65 \mathrm{~g} / \mathrm{cm}^{3}$ de massa específica, $8 \%$ de adesivo e partículas naturais de casca de arroz, valor inferior ao deste estudo $\left(38,44 \mathrm{kgf} / \mathrm{cm}^{2}\right)$, para chapas com características semelhantes.

Analisando os gráficos da Figura 3, nota-se que os valores de MOR ascendem com o aumento da massa específica. Da mesma forma, Haselein, Vital e Dellalucia (1989), Hillig, Haselein e Santini (2002) e Albuquerque e Iwakiri (2005) observaram que o aumento na densidade resulta em aumento no MOR.

Quanto aos três teores de adesivo, foi possível observar que o aumento desta propriedade na chapa, acarretou no acréscimo dos valores de MOR para ambos os tipos de partículas. Comportamento semelhante foi encontrado por Iwakiri et al. (2000), que utilizaram 2 teores de adesivos (8 e 12\%) e avaliaram que, com $12 \%$ de adesivo, era possível obter maiores valores de MOR.

$\mathrm{O}$ teor de adesivo diferenciou estatisticamente (5\% de probabilidade de erro) para as chapas de partículas naturais e processadas. As linhas ajustadas, verificadas na Figura 3, são superiores para maiores porcentagens de adesivos. Comportamento igualmente referido por Keinert Junior e Matos (1987), Brito e Peixoto (2000) e Albuquerque e Iwakiri (2005).

Para chapas de partículas processadas, por exemplo, observaram-se valores mais baixos de MOE com $7 \%$ de adesivo $\left(1115,60\right.$ a $3221,02 \mathrm{kgf} / \mathrm{cm}^{2}$, de 0,65 a $1,15 \mathrm{~g} / \mathrm{cm}^{3}$, respectivamente), que aumentaram com $10 \%\left(2169,60\right.$ a $4064,71 \mathrm{kgf} / \mathrm{cm}^{2}$, de 0,65 a $1,15 \mathrm{~g} / \mathrm{cm}^{3}$, respectivamente) e ainda mais com $13 \%$ $\left(3119,60\right.$ a $8991,60 \mathrm{kgf} / \mathrm{cm}^{2}$, de 0,65 a $1,15 \mathrm{~g} / \mathrm{cm}^{3}$, respectivamente). As chapas de partículas naturais tiveram variação semelhante, sendo a melhor média para MOE de 7005,86 kgf/ $\mathrm{cm}^{2}$. 

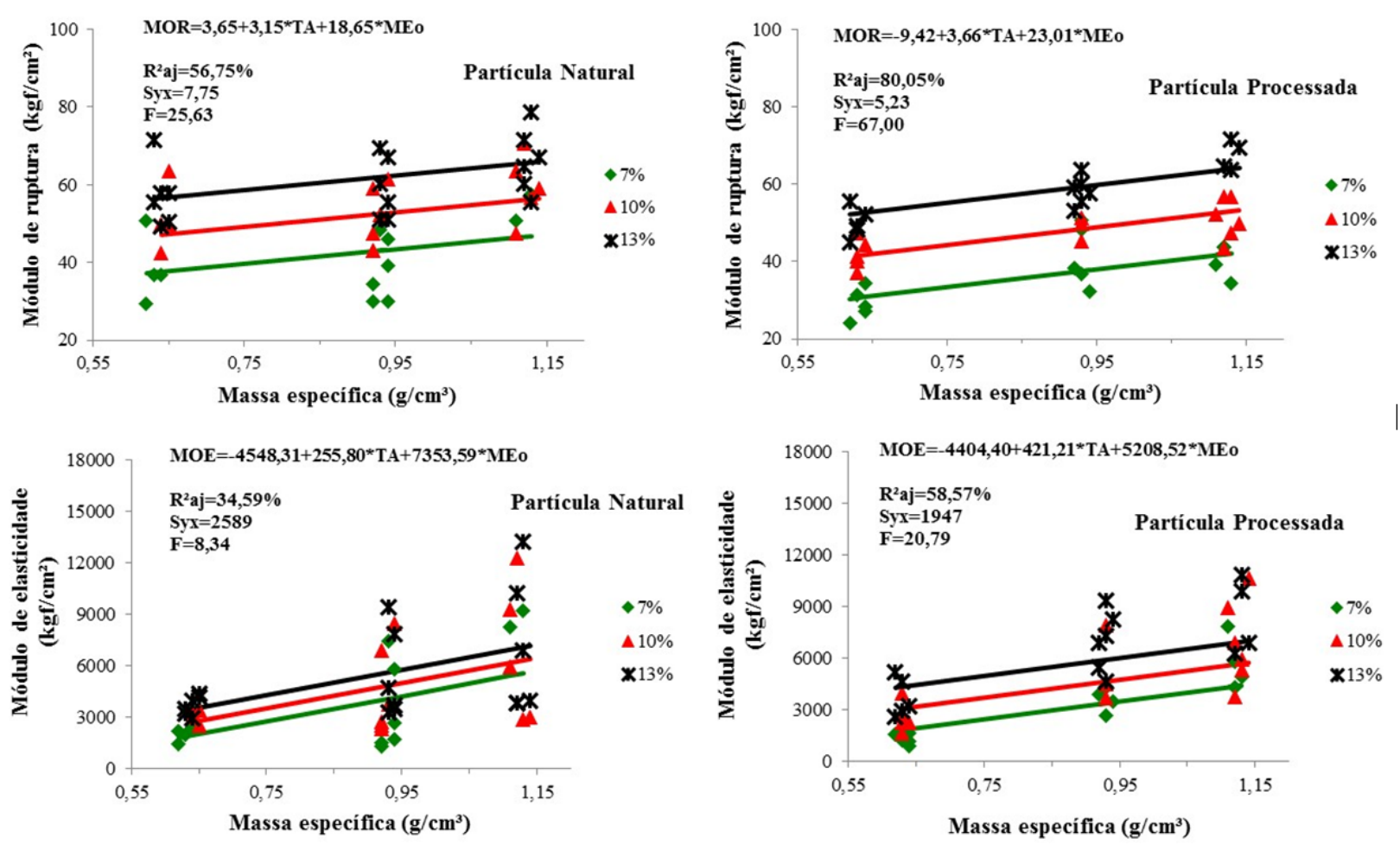

FIGURA 3: Valores de módulo de ruptura e módulo de elasticidade observados e ajustados em função da massa específica, para chapas de partículas naturais e processadas, com 3 teores de adesivos.

FIGURE 3: Values of rupture and elasticity modulus observed and adjusted as a function of density, for particleboard of natural and processed particles, with 3 levels of adhesives.

A norma americana de comercialização ANSI A 208.1 (1987) para chapas de média densidade $\left(0,64\right.$ a $\left.0,80 \mathrm{~g} / \mathrm{cm}^{3}\right)$ e alta densidade $\left(0,80\right.$ a $\left.1,12 \mathrm{~g} / \mathrm{cm}^{3}\right)$ do tipo 1 admite como valores mínimos requeridos 17600 e $24480 \mathrm{kgf} / \mathrm{cm}^{2}$, respectivamente. Nesse sentido, pode-se observar que para o MOE nenhum dos tratamentos atingiu os valores requeridos pela normativa.

Embora os painéis de casca de arroz não atendam às normas de comercialização, os valores de MOE são superiores aos encontrados por Sampathrajan, Vijayaraghavan e Swaminathan (1992) com casca de amendoim, Panyakaew e Fotios (2011) com casca de coco e Cravo et al. (2015) com casca de coco e mistura equivalente de casca de coco e amendoim.

Autores que trabalharam com partículas de madeira encontraram médias de MOE superiores. Iwakiri et al. (2000) encontraram valores para o MOE variando de 12422,48 a 23516,71 kgf/ $\mathrm{cm}^{2}$, em chapas de Eucalyptus spp., com $8 \%$ de adesivo, emulsão de parafina, massa específica de $0,75 \mathrm{~g} / \mathrm{cm}^{3}$ e prensagem durante 8 minutos. Pedrazzi et al. (2005), trabalhando com aglomerados de palitos e serragem da espécie Eucalyptus saligna, com massa específica de $0,65 \mathrm{~g} / \mathrm{cm}^{3}, 8 \%$ de adesivo e prensagem durante 8 minutos, encontrou MOE variando entre 9798,53 e 15193,61 kgf $/ \mathrm{cm}^{2}$, respectivamente, para palitos e serragem.

Os resultados alcançados no presente estudo discordam de Hancock e Chandramouli (1974), autores que reportam similaridade entre propriedades de chapas produzidas com casca de arroz e com partículas de madeira. Cabe ressaltar que na confecção dos painéis, se não mensuradas criteriosamente todas as suas etapas, há influência diretamente na qualidade do produto final e, desta maneira, pode ter contribuído na divergência de resultados. 


\section{Arrancamento de parafuso (AP)}

Observou-se que esta propriedade mecânica teve valores superiores em chapas de partículas naturais para $13 \%$ de adesivo, já para 7 e $10 \%$ os valores ora eram maiores para partículas naturais, ora para processadas. Estas médias variaram de 12,33 kgf, para 7\% de teor de adesivo, 0,65 de massa específica e partícula processada a $37,33 \mathrm{kgf}$, para $13 \%$ de teor de adesivo, 1,15 de massa específica e partícula natural (Figura 4).

Como era esperado, o arranchamento de parafuso mostrou diferença significativa, a $95 \%$ de confiabilidade, nas três classes de massa específica. A linha ajustada do modelo foi crescente com o aumento dessa variável, para ambas as partículas. Portanto, de modo geral, quanto maiores os valores de massa especifica, maior a resistência, o que está de acordo com Moslemi (1974). Este comportamento pode ser facilmente explicado pela diminuição dos espaços vazios em amostras mais densas.

As chapas de partículas naturais e processadas, com coeficiente de determinação de 66,32 e 73,97\%, respectivamente, tiveram diferença significativa a $5 \%$ de probabilidade de erro, quanto ao teor de adesivos. De maneira geral, quanto maior o teor de adesivo, maior a resistência ao arranchamento de parafuso. Além disso, visualizando a Figura 4, percebe-se que as retas se distanciam com esse aumento, ou seja, com 13\% de adesivo ocorre uma maior diferença de resistência do que a 10 e 7\%. Possivelmente, a massa específica pode ter influenciado neste comportamento.

Os resultados encontrados para o arranchamento, independentemente das classes de massa específica, foram inferiores aos exigidos pela norma ANSI A 208.1 (1987) para chapas de média densidade $\left(0,64\right.$ a $\left.0,80 \mathrm{~g} / \mathrm{cm}^{3}\right)$, que admitem um valor mínimo requerido de $102 \mathrm{kgf}$.

Dacosta et al. (2005), em chapas constituídas por maravalha de Pinus elliottii e massa específica de 0,60 e $0,70 \mathrm{~g} / \mathrm{cm}^{3}$ com $8 \%$ de ureia-formaldeído, observaram 55 e $75 \mathrm{kgf}$, respectivamente, valores superiores aos do presente estudo. Calegari et al. (2004) avaliaram painéis de Pinus elliottii com $0,65 \mathrm{~g} / \mathrm{cm}^{3}$ de massa específica e $10 \%$ de teor de adesivo e obtiveram $105 \mathrm{kgf}$, valor também superior ao do presente estudo. Melo (2009) explica que a superioridade de resistência destas chapas à base de madeira se deve ao fato de que o acréscimo de casca de arroz proporciona uma redução da resistência ao arrancamento de parafuso.
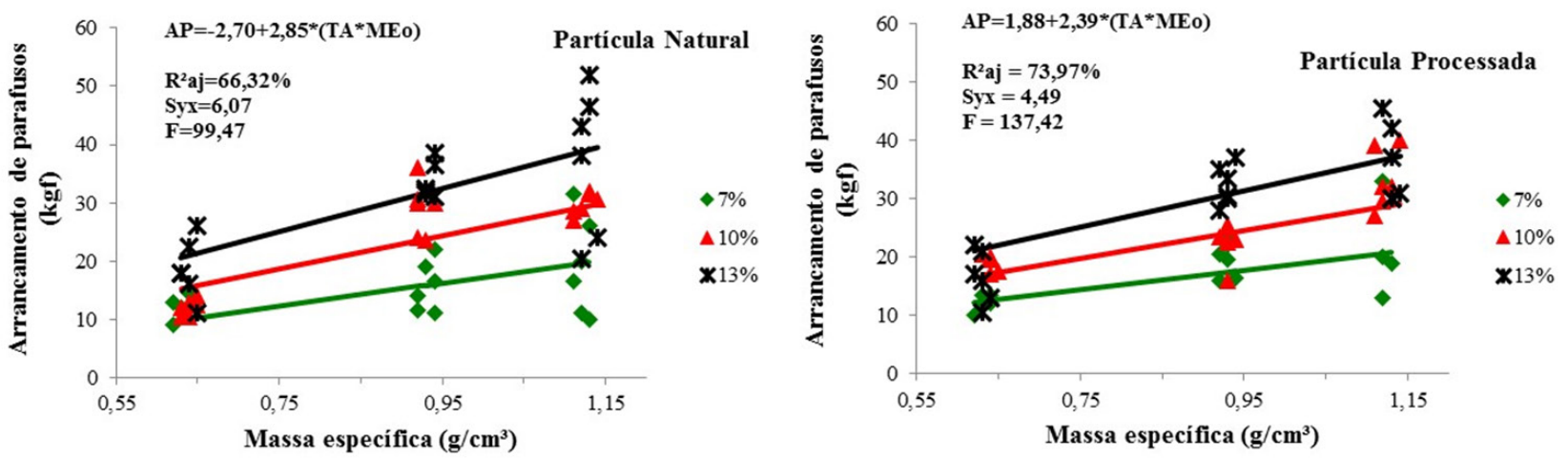

FIGURA 4: Valores de arrancamento de parafusos observados e ajustados em função da massa específica, para chapas de partículas naturais e processadas, com 3 teores de adesivos.

FIGURE 4: Values of screw withdrawal observed and adjusted as a function of density, for particleboard of natural and processed particles, with 3 levels of adhesives. 


\section{Ligação interna (LI)}

Contrário a todos os comportamentos observados até o presente momento, tanto para as propriedades mecânicas quanto para a maioria das propriedades físicas, a ligação interna não obteve alteração significativa (95\% de confiabilidade) nos seus valores, permanecendo constante com o aumento da massa específica (Figura 5).

Resultado contrário ao que a literatura tem se deparado (HASELEIN; VITAL; DELLALUCIA, 1989). Estes autores descreveram que chapas mais densas apresentaram valores mais altos de ligação interna. Afirmam ainda que uma maior densificação proporciona a diminuição de espaços vazios e da porosidade, resultando, desse modo, em uma colagem mais eficiente. Entretanto, os autores relatam outro fator de reconhecida influência sobre esse parâmetro, que é a quantidade e a qualidade do adesivo utilizado na manufatura das chapas. Iwakiri et al. (2004) acrescentaram que essa diferença de comportamento pode ser atribuída à menor disponibilidade de adesivo em função do aumento da quantidade de partículas. Esta menor disponibilidade, muitas vezes, contribui para o não aumento desta propriedade mecânica com o acréscimo da ME.

Do mesmo modo que nas demais propriedades mecânicas, ocorreu diferença significativa a 5\% de probabilidade de erro para a ligação interna nos três teores de adesivos analisados, estabelecendo uma relação crescente entre essa variável e o teor de adesivo. Resultando similaridade com Brito e Peixoto (2000), Iwakiri et al. (2000), Maciel (2004), Dacosta et al. (2005), Iwakiri et al. (2004), Pedrazzi et al. (2005) e Colli et al. (2010).

Os valores médios encontrados para ligação interna em chapas de partículas naturais e processadas variaram de $0,08 \mathrm{kgf} / \mathrm{cm}^{2}$ para $7 \%$ de adesivo a $0,47 \mathrm{kgf} / \mathrm{cm}^{2}$ para $13 \%$ de adesivo. As normas de comercialização americana (ANSI A 208.1, 1987) e alemã (DIN 68761, 1961) estabelecem os valores mínimos de 4,22 e 3,50 kgf/ $/ \mathrm{cm}^{2}$, respectivamente. Sendo assim, as médias atingidas ficaram notavelmente abaixo do mínimo exigido pelas normas referenciadas.

Resultados inferiores também foram observados por Hiziroglu, Jarusombuti e Fueangvivat (2005) para chapas de Eucalyptus camaldulensis com palha de arroz, Pauleski et al. (2007) em compósitos manufaturados com quatro lâminas de polietileno de alta densidade (PEAD), 100, 75, 50, 25 e 0\% de casca de arroz e madeira, Araújo et al. (2014) em painéis MDP com casca de café e partículas de bambu, Lee et al. (2003) com farelo de casca de arroz consorciada à madeira e Panyakaew e Fotios (2011) com casca de coco.

Ndazi et al. (2006) produziram aglomerados com casca de arroz e adesivo à base de tanino de
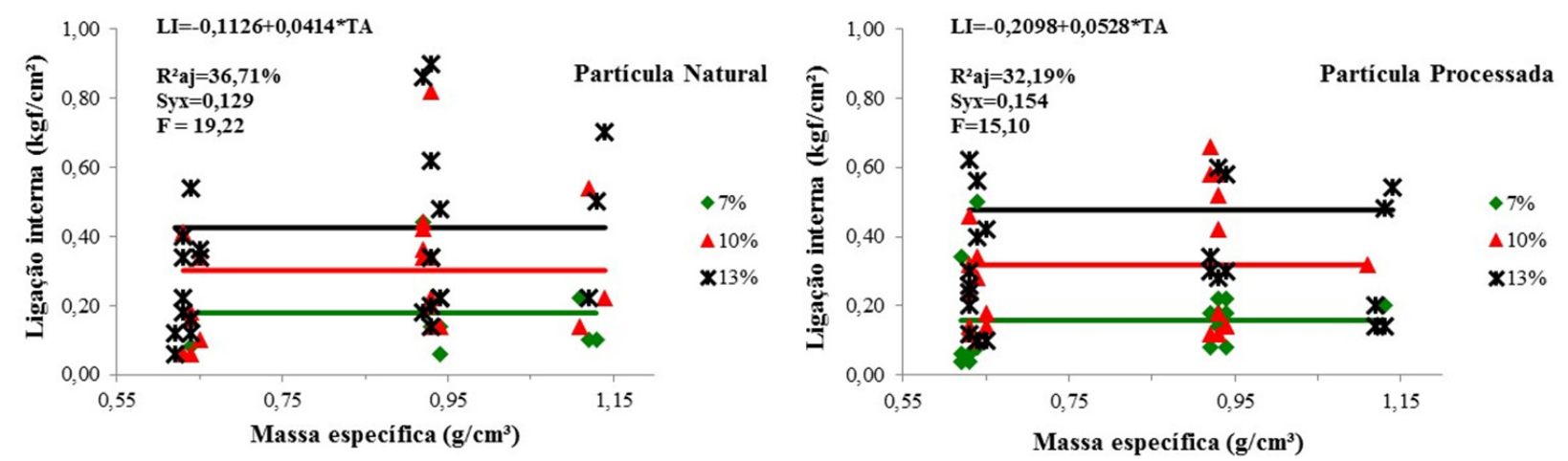

FIGURA 5: Valores de ligação interna observados e ajustados em função da massa específica, para chapas de partículas naturais e processadas, com 3 teores de adesivos.

FIGURE 5: Values of internal bonding observed and adjusted as a function of density, for particleboard of natural and processed particles, with 3 levels of adhesives. 
acácia mimosa. Segundo os autores, a casca de arroz está entre os resíduos agrícolas que estão disponíveis facilmente em grandes quantidades e, nesse ponto de vista, pode ser considerada uma excelente matériaprima para a produção de chapas. A maior dificuldade está na falta de interação direta com a maioria dos adesivos.

Conhecendo essa dificuldade de colagem apresentada pelas partículas de casca de arroz, Hse e Choong (2002) conduziram estudos para desenvolver um adesivo efetivo e econômico para melhorar as propriedades físico-mecânicas de chapas de casca de arroz, em que foram avaliados três adesivos: ureiaformaldeído, fenol-formaldeído e di-isocianato em várias formulações. Os autores chegaram à conclusão que as chapas coladas com 1\% MDI mais $6 \%$ UF apresentaram os melhores resultados quanto à ligação interna, MOE, MOR e estabilidade dimensional.

\section{CONCLUSÕES}

Com o aumento da massa específica e do teor de adesivo as chapas apresentaram menor absorção d'água e inchamento em espessura, após 2 e 24 horas. A exceção foi o inchamento em 2 e 24 horas, para partículas processadas, em que não ocorreu variação com o aumento da massa específica. Somente as chapas com $13 \%$ de adesivo apresentaram valores mínimos requeridos pelas normas de comercialização, quanto à estabilidade dimensional.

As propriedades mecânicas analisadas no presente estudo tiveram valores médios inferiores às normas de comercialização. Entretanto, ficou evidente que o acréscimo da massa específica e do teor de adesivo proporcionou maiores valores para essas propriedades. Com exceção da ligação interna, que permaneceu constante com a ampliação da massa específica.

A utilização da casca de arroz, em comparação com a literatura de chapas com partículas de madeira, reduziu as propriedades fisico-mecânicas que identificam a qualidade dos aglomerados, não sendo recomendados como material estrutural para indústria moveleira e construção civil. Contudo, estes poderiam ser indicados para aplicações que não exijam elevada solicitação, como por exemplo, isolante térmico acústico, decoração, pequenos objetos e artesanato. No entanto, para uma recomendação mais criteriosa, ensaios específicos que avaliam esses parâmetros devem ser realizados.

Nos próximos estudos, para produção de chapas com características melhoradas, recomenda-se a adição de partículas de madeira juntamente à casca de arroz.

\section{REFERÊNCIAS}

ALBUQUERQUE, C. E. C.; IWAKIRI, S. Efeito da adição de água na evolução da temperatura no miolo de aglomerados durante o ciclo de prensagem. Floresta, Curitiba, v. 35, n.1, p. 57-67, 2005.

AMERICANNATIONALSTANDARDS INSTITUTE. ANSI-A-208.1-87. Mat-formed wood particleboard. New York, 1987.

AMERICAN SOCIETY FOR TESTING AND MATERIALS. ASTM D 1037. Standard test methods for evaluating properties of Wood based fiber and particle panel materials. Philadelphia: ASTM, 1998.

ARAÚJO, I. I. et al. Aproveitamento do resíduo do processamento do café na composição de painéis MDP. In: WORKSHOP DO PGR EM GESTÃO DE RESÍDUOS DA UNESP/ANNUAL MEETING, 3., 2014. Proceedings... Archives of health investigation, v. 3, n. spec 2, p. 30-33, 2014.

ASSOCIAÇÃO BRASILEIRA DE NORMAS TÉCNICAS. NBR-14810-1: Chapas de madeira aglomerada. 2. ed. Rio de Janeiro, 2006. 51 p.

BRITO, E. O.; PEIXOTO, G. L. Avaliação da granulometria de partículas de Pinus taeda combinadas com adesivos comerciais para a fabricação de aglomerados. Floresta e Ambiente, Seropédica, v. 7, n. 1, p. 60-67, 2000.

CALEGARI, L. et al. Adição de aparas de papel reciclável na fabricação de chapas de madeira aglomerada. Ciência Florestal, Santa Maria, v. 14, n. 1, p. 193-204, 2004.

CARNEIRO, A. C. O. et al. Propriedades de chapas de aglomerado fabricadas com adesivo tânico de angico-vermelho (Anadenanthera peregrina) e uréia-formaldeído. Revista Árvore, Viçosa, MG, v. 33, n. 3, p. 521-531, 2009. 
COLLI, A. et al. Propriedades de chapas fabricadas com partículas de madeira de Paricá (Schyzolobium amazonicum Huber ex. Ducke) e fibras de Coco (Cocos nucifera L.). Revista Árvore, Viçosa, MG, v. 34, n. 2, p. 333-338, 2010.

CRAVO, J. C. M. et al. Painel aglomerado de resíduos agroindustriais. Ciência Florestal, Santa Maria, v. 25, n. 3, p. 721-730, 2015.

DACOSTA, L. P. E. et al. Propriedades físicas de chapas de partículas aglomeradas fabricadas com resíduos do processamento mecânico da madeira de Pinus elliotii Engelm. Ciência Florestal, Santa Maria, v. 15, n. 4, p. 421-429, 2005.

GERMAN STANDARDS COMMITTEE. Deutschen Normanausschuss. DIN 68761 (1)-1961 (3). Specifications for particleboards. Holtz, 1961.

HANCOCK, W. V.; CHANDRAMOULI, P. Comparatives proprieties of Rice husk board, particleboard, and wafer board. Journal of the Indian Academy of Wood Science, Indian, v. 5, n. 1, p. 18-27, 1974.

HASELEIN, C. R.; VITAL, B. R.; DELlALUCIA, R. M. Fabricação de chapas de aglomerado com madeiras de eucalipto (Eucalyptus grandis (W. Hill ex-Maiden) e de embaúba (Cecropia sp). Revista Árvore, Viçosa, MG, v. 13, n. 2, p. 134-145, 1989.

HILLIG, E.; HASELEIN, C. R.; SANTINI, E. J. Propriedades mecânicas de chapas aglomeradas estruturais fabricadas com madeiras de pinus, eucalipto e acácia-negra. Ciência Florestal, Santa Maria, v. 12, n. 1, p. 59-70, 2002.

HIZIROGLU, S.; JARUSOMBUTI, S.; FUEANGVIVAT, V. Properties of bamboo-rice straw-eucalyptus composite panels. Forest Products Journal, Madison, v. 55, n. 12, p. 221-225, 2005.

HSE, C. Y.; CHOONG, E. T. Modified formaldehyde based resin adhesives for rice hull wood particleboard. Southern Research Station, Asheville, n. 7, p. 81-86, 2002.

INSTITUTO RIO GRANDENSE DO ARROZ. Informativo IRGA. 2015. Disponível em: $<$ http://irga.rs.gov.br>. Acesso em: 6 dez. 2015.

IWAKIRI, S. et al. Mistura de espécies na produção de chapas de partículas estruturais "waferboard". Revista do Setor de Ciências Agrárias, Curitiba, v. 14, n. 1-2, p. 107-114, 1995.

IWAKIRI, S. et al. Produção de chapas de partículas de madeira aglomerada de Pinus elliottii (Engelm) e Eucalyptus dunnii (Maid). Revista do Setor de Ciências Agrárias, Curitiba, v. 15, n. 1, p. 33-41, 1996.

IWAKIRI, S. et al. Resíduos de serrarias na produção de painéis de madeira aglomerada de eucalipto. Revista Scientia Agraria, Piracicaba, v. 1, n. 1-2, p. 23-28, 2000.

IWAKIRI, S. et al. Produção de painéis de madeira aglomerada de Grevillea robusta A. Cunn. ex R. Br. Revista Árvore, Viçosa, MG, v. 28, n. 6, p. 883-887, 2004.

KEINERT JUNIOR, S.; MATOS, J. L. M. Utilização de Pinus pinaster para a fabricação de chapas de partículas. Floresta, Curitiba, v. 17, n. 1/2., p. 113-120, 1987.

LEE, Y. K. et al. Mechanical properties of rice husk flour-wood particleboard by urea-formaldehyde resin. Journal of the Korean Wood Science and Technology, Republic of Korea, v. 31, n. 3, p. 42-49, 2003.

MACIEL, A. S. et al. Painéis de partículas aglomeradas de Pinus elliottii Engelm., poliestireno (PS) e polietileno tereftatalato (PET). Revista Árvore, Viçosa, MG, v. 28, n. 2, p. 257-266, 2004.

MELO, R. R. et al. Propriedades físico-mecânicas de painéis aglomerados produzidos com diferentes proporções de madeira e casca de arroz. Ciência Florestal, Santa Maria, v. 19, n. 4, p. 449-460, 2009.

MORAIS, W. W. C. Propriedades físico-mecânicas de chapas aglomeradas produzidas com bambu, pinus e eucalipto. 2011. 132 f. Dissertação (Mestrado em Engenharia Florestal) - Universidade Federal de Santa Maria, Santa Maria, 2011.

MOSLEMI, A. A. Particleboard. Carbondale: Illinois University Press, 1974. v. 2.

MENDES, R. F. et al. Painéis aglomerados com bagaço de cana em associação com madeira de eucalipto.

Scientia Forestalis, Piracicaba, v. 38, n. 86, p. 285-295, 2010.

NDAZI, B. et al. Production of rice husks composites with Acacia mimosa tannin-based resin. Journal of Materials Science. London, v. 41, p. 6978-6983, 2006.

PEDRAZZI, C. et al. Qualidade de chapas de partículas de madeira aglomerada fabricada com resíduos de uma indústria de celulose. Ciência Florestal, Santa Maria, v. 16, p. 201-212, 2005.

PIZZI, A.; SCHARFETTER, H.; KES, E. W. Adhesives and techniques open new possibilities for the wood processing industry. 1. Experience with tannin based adhesives. National Timber Research Institute, 
South African, v. 39, n. 3, p. 85-89, 1981.

PANYAKAEW, S.; FOTIOS, S. New thermal insulation boards made from coconut husk and bagasse. Energy and Buildings, Lausanne, Switzerland, v. 43, n. 7, p. 1732-1739, 2011.

PAULESKI, D. T. et al. Características de compósitos laminados manufaturados com polietileno de alta densidade (PEAD) e diferentes proporções de casca de arroz e partículas de madeira. Ciência Florestal, Santa Maria, v. 17, n. 2, p. 157-170, 2007.

SAMPATHRAJAN, A.; VIJAYARAGHAVAN, N. C.; SWAMINATHAN, K. R. Mechanical and thermal properties of particle boards made from farm residues. Bioresource Technology, Netherlands, v. 40, p. 249-251, 1992.

SOCIEDADE SUL BRASILEIRA DE ARROZ IRRIGADO. Arroz irrigado: recomendações técnicas da pesquisa para o Sul do Brasil. [s. 1.]: Pallotti, 2014. 192 p.

VARANDA, L. D. et al. Oat Hulls as addition to high density panels production. Materials Research, São Carlos, v. 16, p. 1355-1361, 2013. 